\title{
Non-Empirical Identification of Trigger Sites in Image Data using Persistent Homology: Crack Formation during Heterogeneous Reduction of Iron-Ore Sinters
}

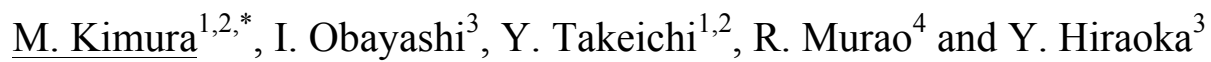 \\ 1. Photon Factory, Institute of Materials Structure Science, High Energy Accelerator Research \\ Organization, Tsukuba, Japan \\ 2. Department of Materials Structure Science, School of High Energy Accelerator Science, SOKENDAI \\ (The Graduate University for Advanced Studies), Tsukuba, Japan \\ 3. Advanced Institute for Materials Research (AIMR), Tohoku University, Sendai, Japan \\ 4. Advanced Technology Research Laboratories, Nippon Steel and Sumitomo Metal Corp., Futtsu, Japan \\ *Corresponding author, email_masao.kimura@kek.jp
}

Trigger sites are specific regions or features of heterogeneity in a material system where key reactions initiate and occur. Previous studies have attempted to determine the locations of trigger sites in image data using various microscopes based on knowledge of materials science ("empirical" approach). These empirical approaches have successfully identified trigger sites in simple systems such as metals. However, these approaches have not proved successful in more complicated systems such as composite materials (e.g., iron ore sinters and carbon fiber reinforced plastics (CFRP)), batteries, and catalysts. Here, we report on our success of a "non-empirical" identification of trigger sites in crack formation during heterogeneous reduction of iron-ore sinters using persistent homology $[1,2]$.

Sinter specimens were prepared by liquid sintering from iron ore and limestone. The specimens were then heated to $1473 \mathrm{~K}$ in reductive gas for reduction, thus simulating the iron-making process. Chemical state mapping was conducted using XAFS at the synchrotron undulator beamline BL-15A1 of the Photon Factory, IMSS, KEK in Japan [3]. The crack formation and phase mapping of larger volumes were investigated using X-CT measurements.

Mapping the valence states of iron oxidation revealed the heterogeneous dynamic evolution of the chemical states from $\mathrm{Fe}$ (III) to Fe(II) during the reduction process (Fig. 1). At an intermediate stage of the reduction process, the spatial distribution of the changes in the reduced areas was heterogeneous rather than homogeneous, resulting in an increase in the local stress and then crack formation. The change in the microstructure (i.e., the heterogeneity of the phase mapping) is very complicated, and we could not determine how the progress of heterogeneous reduction causes crack formation nor empirically identify trigger sites.

However, we identified the most representative topological features that characterize the reduction process using persistent homology and the trigger sites for crack formation using machine-learning techniques. The X-CT datasets of the reduced sinters were deconvoluted into (a) the initial pores, (b) the microcracks formed during reduction, (c) calcium-ferrite phases, and (d) iron-oxide phases. Our analysis using persistent homology involved (1) transforming each image into a persistence diagram (PD) and then (2) into a vector, (3) feeding the vectors together with the measured crack areas into the absolute shrinkage and selection operator (LASSO), (4) identifying the dominant birth-death pairs, and finally (5) mapping them back into the original image. 
Four types of trigger sites, "hourglass"-shaped calcium ferrites (narrow and wide) and "island"- and "hourglass"-shaped iron oxides, were determined to initiate crack formation using only mapping data depicting the heterogeneities of phases and cracks without prior mechanistic information (Fig. 2). These triggers sites, particularly "island" types, were rather difficult to predict empirically, although the determined trigger sites were reasonable in terms of materials-scientific knowledge.

We propose a new approach to identify trigger sites in order to determine macroscopic properties in cases when heterogeneous reactions progress microscopically [1]. The identification of these trigger sites can provide a design rule for reducing mechanical degradation during the reduction process. Furthermore, this approach is expected to deal with multi-dimensional data obtained by spectroscopic imaging techniques such as TXM (Transmission X-ray Microscopy) and STXM (Scanning TXM).

Experiments using synchrotron radiation were performed with the approval of the Photon Factory at IMSS, KEK Program Advisory Committee (Proposal Nos. 2014G707, 2015S2-002, and 2016S2-001). The parts of the study performed by M.K., I.O., Y.T., and Y.H. were supported by the Structural Materials for Innovation of the Cross-ministerial Strategic Innovation Promotion Program (SIP) of Japan Science and Technology Agency (JST): units D66 (M.K., Y.T.) and D72 (I.O., Y.H.).

\section{References:}

[1] M Kimura et al, Scientific Reports 8 (2018) 3553.

[2] I Obayashi et al, J. App. and Comp. Topology (2018) (accepted) (doi.org/10.1007/s41468-018-0013-5).

[3] M Kimura et al, Journal of Physics: Conference Series 849 (2017) 012015.
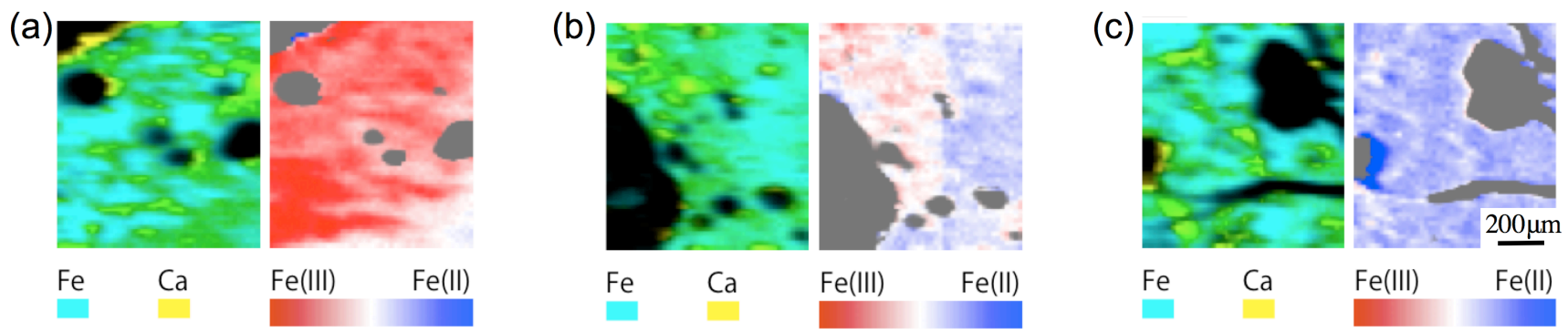

Figure. 1. Right panel: chemical state mapping of an iron ore sinter determined by XANE. The fraction ratio of $\mathrm{Fe}(\mathrm{II}) /(\mathrm{Fe}(\mathrm{II})+\mathrm{Fe}(\mathrm{III}))$ is color-coded: blue for 1 and red for 0 . (a) Early, (b) Intermediate, and (c) Final stages of reduction. Left panel: the elemental distribution of iron (blue) and calcium (yellow).

(a)

Figure. 2. Results of persistent homology and machine learning of the image data. (a) Four types of trigger sites that were non-empirically identified by the new approach. (b) Cracks formed that were measured by X-CT.

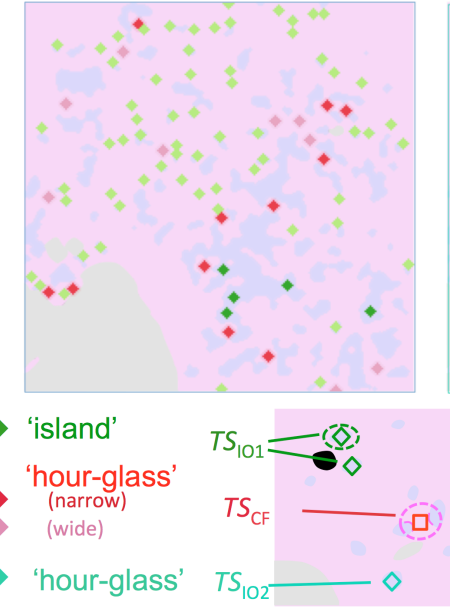

(b)

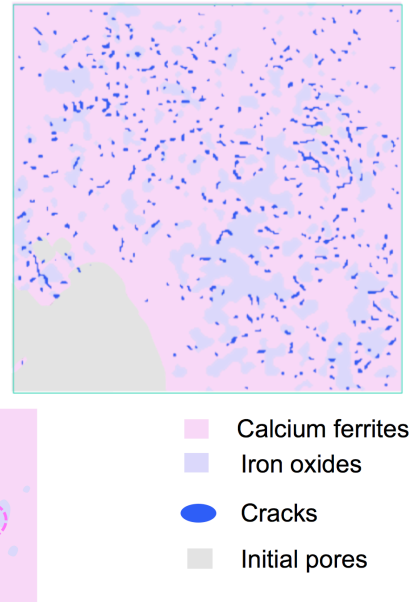

